\title{
PREENCHIMENTO DA CADERNETA DE SAÚDE DA CRIANÇA NA VISÃO DE TÉCNICOS DE ENFERMAGEM
}

\section{Carine de Pelegrini Bissacot ${ }^{1}$;Giovana Luiza Rossato²; Mariana Fogaça Martin ${ }^{3}$; Mateus Celio da Silva ${ }^{4}$; Silvana Leão ${ }^{5}$; Rosiane Filipin Rangel ${ }^{6}$ \\ RESUMO}

Objetivou-se conhecer a visão dos técnicos de enfermagem no preenchimento da caderneta de saúde da criança. Trata-se de uma pesquisa-ação. Fizeram parte da pesquisa dezesseis técnicos de enfermagem que desenvolvem a assistência à criança nas Equipes de Atenção Primária e Estratégias de Saúde da Família, em um município da região central do estado do Rio Grande do Sul. Os dados foram coletados por meio de um questionário online no Google Forms, analisados de acordo com Análise Textual discursiva. No presente estudo, são apresentados os dados da primeira fase da pesquisa. Emergiram duas categorias: Potencialidades evidenciadas por técnicos de enfermagem no preenchimento da Caderneta de Saúde da Criança; Fragilidades evidenciadas por técnicos de enfermagem no preenchimento da Caderneta de Saúde da Criança. Evidenciou-se através da análise das falas que, embora existam fragilidades, o documento é extremamente importante para a prevenção e promoção da saúde.

Palavras-chave: Assistência integral à saúde da criança; Desenvolvimento infantil; Enfermagem; Saúde da criança;

Eixo Temático: Atenção Integral e Promoção à Saúde (AIPS)

\section{INTRODUÇÃO}

A caderneta de saúde da criança (CSC) ou passaporte da cidadania contém informações referentes ao desenvolvimento saudável, direitos e deveres das crianças e dos pais, aleitamento materno, vacinas, saúde bucal, alimentação

\footnotetext{
${ }^{1}$ Acadêmica do Curso de Enfermagem - Universidade Franciscana; E-mail- cabissacot@gmail.com

2 Acadêmica do Curso de Enfermagem - Universidade Franciscana (UFN); E-mail giovana.rossato@ufn.edu.br

${ }^{3}$ Acadêmica do Curso de Enfermagem - Universidade Franciscana (UFN); E-mail mariana.fmartins@ufn.edu.br

4 Acadêmico do Curso de Medicina - Universidade Franciscana (UFN); E-mail mateusceliodasilva@gmail.com

5 Acadêmica do Curso de Enfermagem - Universidade Franciscana (UFN); E-mail silvana.d.leao@gmail.com

6 Orientadora. Enfermeira. Doutora em Enfermagem. Docente do curso de Enfermagem Universidade Franciscana (UFN); E-mail rosiane@ufn.edu.br
} 
complementar, marcos de desenvolvimento, entre outros assuntos que são de extrema relevância no cuidado em cada fase da infância (BRASIL, 2020a).

A CSC passou por reformulações que devem ser do conhecimento de todos os profissionais que prestam assistência a esse público, dentre esses os técnicos de enfermagem. Considera-se a importância desse profissional no cuidado, pois é parte da equipe de atenção primária, que possui como umas de suas atribuições garantir a atenção à saúde das pessoas com vistas a um cuidado integral e, geralmente realizam as primeiras avaliações e identificação das necessidades, vulnerabilidades e intervenções precoces (BRASIL, 2012)

Nessa direção, Silva, Gaíva e Mello (2015) reforçam que a CSC é um documento no qual se preconiza a vigilância integral, sendo utilizada pelos profissionais que prestam assistência à criança nos diferentes âmbitos de saúde. Assim, é necessário ter conhecimento de seu conteúdo, bem como de realizá-lo corretamente. Ressalta-se que a utilização e/ou o preenchimento incorreto do instrumento tende a prejudicar a continuidade do cuidado integral da criança em diferentes pontos de atenção, gerando um impacto nos indicadores de saúde infantil (BRASIL, 2020a).

Nesse contexto, objetiva-se com a pesquisa, conhecer a visão dos técnicos de enfermagem no preenchimento da caderneta de saúde da criança. Utilizou-se como questão norteadora do presente estudo: "Qual a visão do técnico de enfermagem referente ao preenchimento da caderneta de saúde da criança?”.

\section{METODOLOGIA}

Esse estudo faz parte de um projeto maior intitulado "Qualificação do Acompanhamento Multiprofissional de Saúde em Relação ao Crescimento e Desenvolvimento Infantil na Região Central do Rio Grande do Sul", aprovado pela chamada Decit/SCTIE/MS-CNPq-FAPERGS n0 08/2020, Programa Pesquisa para o SUS: Gestão compartilhada em saúde - PPSUS. 
Trata-se de uma pesquisa-ação. Esse é um tipo de pesquisa interpretativa que abarca um processo metodológico empírico. Compreende a identificação do problema dentro de um contexto social e/ou institucional, o levantamento de dados relativos ao problema, à análise e significação dos dados levantados pelos participantes, a identificação da necessidade de mudança, o levantamento de possíveis soluções e por fim, a intervenção e/ou ação propriamente dita no sentido de aliar pesquisa e ação, simultaneamente. A pesquisa-ação intervém na prática no sentido de provocar a transformação (KOERICH et al., 2009). Assim, no presente artigo, são apresentados os dados da primeira etapa do estudo.

Participaram da pesquisa 104 atuantes em Equipes de Atenção Primária (EAP) e Estratégias de Saúde da Família (ESF) que desenvolvem assistência à criança, em um município da região central do estado do Rio Grande do Sul, entre os meses de abril a junho de 2021. Para a coleta dos dados, inicialmente, foi solicitado ao Núcleo de Educação Permanente em Saúde (NEPES) o contato das unidades e e-mail dos profissionais de saúde. Na sequência, encaminhou-se via CONSULFARMA e e-mail o convite aos profissionais para participarem da pesquisa. Aqueles que aceitaram, assinaram o Termo de Consentimento Livre e Esclarecido (TCLE) e, após responderam a um questionário online do Google Forms com perguntas abertas e fechadas acerca da temática.

Utilizou-se como critério de inclusão estar desenvolvendo atividades assistenciais no cuidado à criança e de exclusão os profissionais de saúde que estavam em laudo, afastamentos, ou exerciam apenas cargos gerenciais ou de gestão. Os dados foram analisados conforme a Análise Textual Discursiva, na qual a análise se inicia junto à coleta de dados, consistindo num processo integrado de análise e de síntese, baseado em uma leitura rigorosa e aprofundada dos textos, descrevendo e interpretando fenômenos e discursos (MORAES; GALIAZZI, 2011). As questões éticas foram respeitadas de acordo com a Resolução CNS n 466/12. $O$ projeto foi aprovado pelo Comitê de Ética da Universidade Franciscana - UFN sob o $\mathrm{n}^{\circ}$ 4.364.999. Visando manter o sigilo e anonimato, os profissionais serão 
EDUGAÇÃO, SAÚDE

ETECNOLOGIA

26 A 28 DE OUTUBRO DE 2021

identificados pela TE (técnico de enfermagem), seguida de número cardinal (1, $2,3 \ldots)$.

\section{RESULTADOS E DISCUSSÕES}

Dos dados organizados e analisados se elencou duas categorias: Potencialidades evidenciadas por técnicos de enfermagem no preenchimento da CSC; Fragilidades evidenciadas por técnicos de enfermagem no preenchimento da CSC. Nos resultados evidenciaram-se potencialidades destacadas pelos profissionais, como o fácil entendimento e os benefícios para a vigilância do público infantil, bem como as fragilidades relatadas como falta de capacitação e preenchimento inadequado.

\section{Potencialidades evidenciadas por técnicos de enfermagem no preenchimento da Caderneta de Saúde da Criança.}

A CSC visa o desenvolvimento e a promoção de saúde, através de seu uso correto, torna-se possível acompanhar a base do crescimento e progresso da criança, como também seus dados de identificação. Ademais, esse documento transcende o objetivo de apenas acompanhar o crescimento, pois possui conteúdos que estimulam um maior vínculo mãe-filho, como orientações sobre amamentação e alimentação do recém-nascido. Assim, cumpre o papel de instrumento de comunicação, vigilância, prevenção e promoção da saúde da criança (SBP, 2017).

No decorrer dos questionamentos percebeu-se a importância e os benefícios da CSC para os profissionais, pois por meio dela obtêm-se informações relevantes para o melhor acompanhamento infantil e para que se torne possível uma efetiva promoção da saúde. Conforme evidenciado nas falas a seguir:

"A caderneta da criança é rica em informações, devemos preencher corretamente" (TE 13). 
“A caderneta, em si, é ótima aliada para o acompanhamento infantil" (TE 15).

A CSC possibilita que o profissional acompanhe o crescimento e a evolução na infância, considerando estratégias para a sua melhor qualidade de vida e ampliando as informações de saúde. Neste contexto, adquire-se como benefícios o controle de doenças preveníveis por imunização, a inclusão de conteúdos adicionais sobre gravidez, parto e puerpério, orientações em relação a alimentação e amamentação, bem como espaços para anotações de dados relevantes em relação ao paciente, como estatura e suplementação profilática de ferro (PEDRAZA, 2019).

Cabe ressaltar que, a CSC foi elaborada em 2005 pelo Ministério da Saúde e veio para substituir o Cartão da Criança, sendo um documento mais amplo e completo, agregando gráficos mais aprofundados sobre o crescimento e desenvolvimento, além de incluir os dados acerca da saúde bucal, visual e auditiva e da vacinação, trazendo também orientações mais detalhadas sobre a amamentação e cuidados importantes desde os primeiros dias de vida até o completo desenvolvimento infantil (LIMA et al., 2016).

Sua última atualização, feita em 2020, acoplou ainda mais informações tornando-a um "livreto" informativo; tal característica potencializadora é por vezes vista como uma fragilidade, pois seu grande número de páginas acaba por impactar os profissionais que relatam esta ser muito extensa e repetitiva, podendo tornar o preenchimento superficial. No entanto, apesar do receio em colaborar com o preenchimento completo da caderneta, há de se levar em consideração a sua linguagem acessível e campos de preenchimento de fácil compreensão, assegurando assim um instrumento de alta funcionalidade e manuseio, como evidenciado nas seguintes falas:

"São muito claras as informações contidas nela" (TE 11)

"Bem fácil de ser preenchida” (TE 14). 
Ressalta-se que a primeira parte da caderneta é direcionada a família ou a quem cuida da criança, contendo informações sobre saúde, direitos da criança, vacinação, amamentação e alimentação saudável, entre outros pontos; já a segunda parte destina-se aos profissionais de saúde, contendo gráficos de crescimento, tabela de registro para vacinas, com espaço também para registro de informações importantes. De tal modo, este documento é completo e de caráter otimizador quanto a saúde da criança, além de facilitador do serviço de saúde, pois contém a maioria das informações necessárias e pertinentes no cotidiano dos pais e cuidadores (SILVA; GAÍVA; MELLO, 2015).

\section{Fragilidades evidenciadas por técnicos de enfermagem no preenchimento da Caderneta de Saúde da Criança.}

É atribuição do técnico de enfermagem dentro do serviço de atenção primária à saúde (APS) a realização de atividade para atenção de demanda espontânea, bem como a participação do acolhimento das crianças de maneira a realizar a escuta qualificada e identificação das necessidades de saúde e classificação de risco, avaliação da vulnerabilidade e coleta de informações e sinais clínicos essenciais para constar na caderneta e para o olhar clínico que será direcionado a criança. Além disso, também atividades de educação permanente em saúde e ações de promoção e prevenção de saúde (BRASIL, 2020b).

Nessa direção, foi possível observar nas falas que, há lacunas no que tange ao conhecimento de tarefas e da abrangência multidisciplinar que a CSC possui, muitas vezes ainda sendo vista com o preenchimento exclusivo do médico e do enfermeiro, ou em alguns casos o não entendimento dela.

“Não me sinto capacitada para realizar esse procedimento" (TE16).

"Não preencho" (TE8) 
Percebe-se a necessidade de reflexão e desenvolvimento de estratégias por parte dos gestores que instiguem e possibilitem os profissionais das equipes de saúde compreender a abrangência e importância de conhecer esse documento, não somente para o preenchimento, mas também por conter informações que podem ser utilizadas nas orientações e atividades de promoção e prevenção da saúde com os pais/cuidadores e trabalhadores, que visam a integralidade do cuidado.

A capacitação e posse do que lhe é competência é um grande aliado para a continuidade de cuidado e acompanhamento infantil, de modo que se faz indispensável para a saúde integral da criança. No serviço se constata a não realização do que lhe é competência, consequentemente gerando uma descontinuidade e uma quebra na vigilância infantil. A realização do mesmo é de caráter multidisciplinar a ser implementado no serviço, bem como atividades que visam a educação permanente e promoção e prevenção de agravos, sendo assim parte do trabalho do técnico de enfermagem que deverá realizar com qualidade. $\mathrm{A}$ sua realização é um aliado na identificação precoce de doenças e seu devido encaminhamento a fim de ter a menor repercussão possível na vida futura da criança e nos índices de mortalidade infantil (SILVA; CURSINO; SILVA, 2018).

Conforme consta na Política nacional de atenção integral à saúde da criança (PNAISC) em seu eixo estratégico III do qual se nomeia promoção e acompanhamento do crescimento e desenvolvimento integral, se caracteriza por desenvolvimentos físicos, cognitivos, neuropsicomotores e emocionais, dos quais se inserem vínculos emocionais e interações com o ambiente e pessoas (BRASIL, 2018). Neste contexto, o técnico de enfermagem se faz indispensável como aliado no acompanhamento infantil. Esse profissional, no contexto da atenção primária deve auxiliar nas dúvidas das famílias, orientando quanto ao uso da CSC e preenchendo as partes que o competem.

Outro dado evidenciado nas falas, refere-se ao uso da CSC nas maternidades, visto que, compreende-se como um espaço de alto potencial no que tange as informações relacionadas ao nascimento e o pré-natal, adquirindo-as 
através dos prontuários hospitalares e a Caderneta da Gestante. No entanto, nota-se que o uso inadequado prejudica no acompanhamento infantil em toda rede de saúde, inviabilizando o trabalho dos profissionais no que se refere aos aspectos específicos da mãe e do recém-nascido (ROSOLEM et al., 2019).

"Há falta de anotações importantes, ao nascer, que deixam de ser feitas por quem faz o atendimento durante o período de internação. A carteira, em si, é ótima aliada para o acompanhamento infantil" (TE15).

Evidenciou-se, também, que orientações sobre o uso da caderneta, passadas na maternidade para as famílias, contribuem para que as mesmas sejam utilizadas em outros serviços de saúde, possivelmente usados pela criança, favorecendo, assim, o processo de acompanhamento infantil mediante a corresponsabilização dos pais no que se refere ao controle de saúde dos seus filhos. Com base nisso, as famílias atentam-se no preenchimento adequado da caderneta nos atendimentos de saúde, e desse modo, colaboram na sensibilização dos profissionais de saúde para usá-la como instrumento de monitoração para a saúde infantil (SILVA; CURSINO; SILVA, 2018).

A instabilidade no preenchimento da CSC possui dentre algumas justificativas a falta de capacitação profissional no manuseio e excessivas demandas de obrigações que acarretam no menor tempo de atendimento. Assim, é destacado a presença de dificuldades na compreensão das mudanças sofridas na caderneta ao longo dos anos. Do mesmo modo, que o tempo necessário para tal preenchimento relacionado à intensa rotina de atendimentos, compromete, também, o uso adequado. Na seguinte fala percebe-se que esse descuido no preenchimento pode abranger fatores indiretos, conforme foi exposto (ARAUJO et al., 2021).

"Acredito que muitos profissionais não preenchem as cadernetas por descuido mesmo" (TE11). 
Ademais, as fragilidades captadas no preenchimento da CSC na visão dos técnicos de enfermagem associam-se a diversas dificuldades presentes nos serviços de saúde. No entanto, é percebido por eles que a ocorrência da incompletude da caderneta, e a mesma ocorre por falhas rotineiras. Assim, reconhece-se a necessidade de reflexão por todos os profissionais envolvidos no cuidado, a fim de incentivar as responsabilidades de cada um nos registros da CSC.

\section{CONCLUSÃO}

Evidenciou-se através da análise das falas que embora existam fragilidades, a CSC é extremamente importante para a prevenção e promoção da saúde, pois sua principal potencialidade se dá através de uma visão integral de saúde infantil e na identificação precoce de doenças e atrasos, tendo o técnico de enfermagem como um dos protagonistas de sua inserção e utilização correta no serviço. Constatou-se fragilidades, onde ganham destaques a falta de capacitação profissional referente ao documento e uso inadequado no preenchimento do instrumento.

Nesse sentido, a fim de sanar fragilidades constatadas por técnicos de enfermagem, constatou-se a necessidade para com realizações de capacitações para este grupo de profissionais, com a finalidade de ampliar seus conhecimentos e empoderar do que lhes é competência estar desenvolvendo em seus locais de atuação.

Destaca-se que, por ser parte de uma pesquisa de ação, os dados deste estudo servirão para as capacitações acerca da CSC que serão realizadas com os profissionais no cenário analisado. 


\section{AGRADECIMENTOS}

Agradeço a Fundação de Amparo à Pesquisa do Estado do Rio Grande do Sul FAPERGS pelo financiamento da pesquisa.

\section{REFERÊNCIAS}

ARAUJO M. R. S. et al. Análise dos fatores que podem contribuir para a ausência ou o preenchimento inadequado da caderneta da criança. Revista Eletrônica Acervo Saúde, v. 13, n. 4, p. e6698, 3 abr. 2021.

BRASIL. Secretaria de Atenção à Saúde. Departamento de Atenção Básica. Política Nacional de Atenção Básica / Ministério da Saúde. Secretaria de Atenção à Saúde. Departamento de Atenção Básica. - Brasília. 2012.

BRASIL. Secretaria de Atenção à Saúde Departamento de Ações Programáticas e Estratégicas. Ministério da saúde. Política Nacional de Atenção integral à saúde da criança : Orientação para implementação.Brasília,DF.2018.

BRASIL. Secretaria municipal de saúde. Protocolos de atenção à saúde da criança. Prefeitura Municipal de Nova Lima Dezembro de 2020a.

BRASIL. Governo federal. Ministério da saúde.Caderneta da Criança é ferramenta importante para acompanhamento integral da saúde infantil, 2020b.

MORAES, R.; GALIAZZI, M. C. Análise Textual Discursiva. 2.ed. rev. Ijuí: Editora Unijuí, 2011.

KOERICH, M. S. et al.. Pesquisa-ação: ferramenta metodológica para a pesquisa qualitativa. Rev. Eletr. Enf, Goiânia, v. 11, n. 3, p. 717-723, 2009.

LIMA, L.G. et al. A Utilização da Caderneta de Saúde da Criança no acompanhamento Infantil. Revista Brasileira de Ciências da Saúde v.20 n.2, 2016.

PEDRAZA, D.F. Qualidade do preenchimento do instrumento de acompanhamento da saúde da criança em município do nordeste brasileiro. Revista Gerencia y Políticas de Salud, vol.18, núm.37,2019.

ROSOLEM, L. H. et al. Caderneta de saúde da criança: coordenação do cuidado e acesso à saúde. Cogitare Enfermagem, [S.I.], v. 24, july 2019. ISSN 2176-9133.

SBP. Sociedade Brasileira de Pediatria. Caderneta de Saúde da Criança e do Adolescente: Instrumentos de vigilância e promoção do Desenvolvimento (2017).

SILVA,F.B;GAíVA,M.A.M;MELLO,D.F. Utilização da caderneta de saúde da criança pela família: Uma percepção dos profissionais. Texto Contexto Enferm, Florianópolis, 2015 Abr-Jun; 24(2): 407-14. 
EDUCAÇÃO, SAÚDE

ETECNOLOGIA

26 A 28 DE OUTUBRO DE 2021

QUFN

SILVA,T.C.T; CURSINO,E.G;SILVA,L.F. Caderneta de saúde da criança: Vigilância do crescimento e desenvolvimento infantil. Rev enferm UFPE on line. Recife, 12(12):3445-55, dez., 2018. 